\section{Certificado médico}

\author{
Medical certificate
}

\section{DEFINICIÓN}

El certificado médico es un testimonio escrito acerca del estado de salud actual de un paciente, que el profesional extiende a su solicitud o a la de sus familiares, luego de la debida constatación del mismo a través de la asistencia, examen o reconocimiento.

Existen dos clases de certificado: los obligatorios por ley, entre los que podemos encontrar los de defunción, nacimiento, etcétera, y los simples, que son los que habitualmente se redactan en el consultorio.

Las situaciones que llevan a realizar los certificados simples nos permiten diferenciarlos en:

- Los que acreditan alguna enfermedad que requiera o no baja escolar.

- Los que acreditan aptitud física o psicofísica. Estos a su vez derivan en dos grupos:

a) Los solicitados previamente al comienzo de alguna actividad escolar que requiera la conservación de alguno de los sentidos, cierta capacidad intelectual, movimientos físicos o equilibrio psíquico y cuya ausencia ponga en riesgo al paciente o a terceros.

b) Los solicitados antes de iniciar alguna actividad física, deportiva tanto competitiva como recreativa.

El certificado médico se extiende siempre a petición de la parte interesada, entendiendo por tal al paciente, a la persona a la que el paciente autoriza por escrito o al representante legal. En caso de menores de edad lo harán sus padres o representantes legales.
Armando Reyes-Cadena

Médico adscrito al Departamento de Consulta Externa de pediatría.

Instituto Nacional de Pediatría, México.

Recibido: 18 de febrero del 2015

Aceptado: 31 de marzo del 2015

Correspondencia: Armando Reyes-Cadena Instituto Nacional de Pediatría Insurgentes Sur 3700-C CP 04530 México, D.F.

Tel.: 10-84 09-00 ext. 1832

cadenadr@yahoo.com.mx

Este artículo debe citarse como

Reyes-Cadena A. Certificado médico. Acta Pediatr Mex 2015;36:201-204. 


\section{CARACTERÍSTICAS}

El certificado médico óptimo debe cumplir con las siguientes características:

a) Veraz. El certificado debe ser reflejo fiel e indudable de la comprobación efectuada personalmente por el profesional que lo expide. El médico, ante las autoridades, es testigo de un hecho, la salud del paciente, y el certificado un testimonio del mismo.

b) Legible. Debe estar escrito de puño y letra, con letra clara o con un formato impreso previamente, que permita ser interpretado en su totalidad, evitando abreviaturas, siglas y códigos.

c) Descriptivo. Debe hacerse constar el diagnóstico que motiva la certificación. En los pacientes en quienes a partir del interrogatorio y la exploración física no se pueda llegar al mismo se recomienda hacer una descripción sindromática. De esta manera se evitan las contradicciones de un diagnóstico equivocado.

d) Coherente. La coherencia debe surgir de la comprobación adecuada efectuada personalmente y de un relato de lo observado estrictamente al atender al paciente.

e) Documentado. El testimonio del médico debe quedar documentado en la Historia Clínica o ficha de consultorio, de manera tal que fundamente lo expresado en el mismo $y$, ante una eventual investigación judicial, sustente la veracidad de lo certificado.

f) Limitado. Esta característica se refiere a la necesidad de aclarar en el texto del certificado la actividad para la cual se considera apto el paciente, evitando de esta manera que el certificado sea utilizado con fines para los cuales el paciente no está capacitado. g) Formal. El certificado es un testimonio escrito y requiere un marco acorde con el mismo:

- Utilización de papel membretado: recetario particular o institucional

- Ser de puño y letra del profesional

- Estar firmado y sellado

- Contar con los datos de identificación del paciente: nombre, apellidos, edad, sexo, historia clínica.

- Contar con los datos de expedición: lugar, fecha y hora de expedición del mismo

Objetivos de la consulta al realizar el certificado médico

a) Objetivos primarios. Determinar situaciones que puedan poner en riesgo la salud o la vida de la persona o de terceros y, por consiguiente, contraindicar la actividad para la que se solicita el certificado.

b) Objetivos secundarios. Generar una oportunidad para realizar un control de salud y detectar a tiempo enfermedades hasta el momento no percibidas por el paciente.

Al explorar al paciente se debe intentar aprovechar el encuentro como una oportunidad de control de salud para la implementación de las prácticas de prevención de acuerdo con el sexo, la edad o subgrupo de riesgo, para lo cual será necesario realizar una anamnesis y un examen físico adecuado, evaluando al paciente en forma integral teniendo en cuenta su perfil de riesgo.

Al realizar la anamnesis se debe interrogar al paciente en forma exhaustiva, en el caso de menores deberá hacerse en presencia de los padres y anotar las respuestas en la Historia Clínica. 
En niños en edad escolar se recomienda el examen clínico para realizar certificado médico anualmente con el objetivo de mantener el control de crecimiento y desarrollo; además, para detectar tempranamente factores de riesgo y afecciones prevalentes según la edad y el sexo. Este examen debe constar de:

Interrogatorio. Antecedentes familiares y personales, por ejemplo: muerte súbita en un familiar de primer grado, enfermedades neurológicas, cardiovasculares.

Hábitos. Alimentación, higiene, juego, descanso, etc.

\section{Inmunizaciones.}

Examen físico. Debe ser pertinente, se recomienda realizar una evaluación adecuada para la edad o grupo de riesgo, prestando especial atención a las maniobras que puedan aportar información en relación con el motivo para el cual se va a expedir el certificado médico.

\section{Antropometría. Peso y talla en centiles.}

Examen cardiovascular. Auscultación con el paciente acostado primero y sentado después. Palpación del choque de la punta. Toma de la presión arterial en decúbito y en posición sedente con el manguito adecuado; palpación de los pulsos periféricos comparativamente.

Aparato respiratorio. Auscultación, frecuencia respiratoria; descartar anomalías torácicas; corroborar buena ventilación de vías superiores e inferiores; verificar la salud bucal (caries).

Osteomuscular. Alteraciones posturales, escoliosis; revisar integridad de extremidades inferiores y del arco plantar.
Abdomen. Descartar visceromegalias, hernias.

Sistema nervioso. Evaluar marcha, pruebas de equilibrio y coordinación. Reflejos osteotendinosos. Investigar agudeza visual o auditiva.

Una vez finalizada la anamnesis y el examen físico completo se podrá expedir el certificado médico para la actividad física, pedagógica, recreativa y deportiva escolar. Este certificado debe ser expedido por un médico general, pediatra, médico familiar o por un subespecialista en los casos que lo ameriten.

El certificado médico debe entregarse únicamente al paciente, a la persona que legítimamente lo ha solicitado o a la que haya sido autorizada para ese fin por el paciente.

\section{RECOMENDACIONES ÉTICAS}

Nunca se certificará sobre lo que no se ha observado o comprobado personalmente.

Se debe describir con exactitud y rigor lo constatado, usando para ello expresiones precisas y prudentes.

Se ha de evitar al máximo el empleo de tecnicismos; si ello fuera inevitable se ha de aclarar cuál es su significado para hacerlos comprensibles en lenguaje común.

Es obligado hacer constar la fecha en que se expide el certificado, seguida de la firma del médico que certifica.

Es conveniente dejar en la historia clínica relación de los documentos que se han entregado al paciente, indicando el motivo de haberlos emitido. Es recomendable también guardar una copia de ellos. 


\section{CONCLUSIONES}

El certificado médico es un documento legal al que se le debe dar la importancia que merece.

La solicitud de un certificado médico debe considerarse como una oportunidad para realizar un examen de salud completo.

El médico clínico debe reconocer sus limitaciones como examinador, y no hacer certificaciones en áreas en las que no es competente; pero a la vez lograr la competencia adecuada que le permita no convertirse sólo en una simple guía de derivación.

\section{LECTURAS RECOMENDADAS}

1. Baillieauu N, Arislur G. El Certificado Médico. Actualización en la Práctica Ambulatoria. Mayo-Junio 2006;9(3):8083.

2. Organización Médica Colegial de España. Declaración sobre las cualidades del certificado médico y sobre sus diferencias con los partes y los informes médicos. Declaración aprobada por el Pleno del Consejo general. 26 Enero 2007.

3. El Certificado Médico. Círculo Paraguayo de Médicos. Medicina Pre-paga.

4. Casanovas O, Yulitta H, Turganti A, Lukin A, Medina V, Rubinstein E. Certificación para la actividad física escolar y preparticipativa deportiva. Arch Argen Pediatr 2001;99(6):538-539. 